\title{
Definição de níveis aceitáveis baseados no risco para hidrocarbonetos no Município de Porto Alegre segundo a metodologia RBCA
}

\section{Definition of risk-based screening levels to hydrocarbons in Porto Alegre municipality according to the RBCA methodology}

\author{
Eri Takeuchi \\ Geóloga. Doutoranda do Programa de Pós-Graduação em Geociências da Universidade Federal do Rio Grande do Sul (UFRGS) \\ Ari Roisenberg \\ Geólogo. Professor titular do Instituto de Geociências da Universidade Federal do Rio Grande do Sul (UFRGS)
}

\section{Resumo}

O presente estudo definiu valores de NABR (Níveis Aceitáveis Baseados no Risco) para hidrocarbonetos no Município de Porto Alegre, de acordo a metodologia RBCA, adotando-se dados hidrogeológicos dos compartimentos Embasamento Cristalino alterado e Depósitos Sedimentares Quaternários. Os parâmetros de exposição adequados à população do município, bem como dados de toxicidade dos compostos selecionados, foram definidos a partir de fontes bibliográficas e bancos toxicológicos disponíveis. As tabelas de referência geradas são, em geral, mais restritivas para o Embasamento Cristalino em relação aos Depósitos Sedimentares. Comparados às tabelas de referência da Cetesb, os valores correspondentes a Porto Alegre são inferiores, resultando na necessidade de investigações ambientais mais detalhadas em áreas impactadas por hidrocarbonetos.

Palavras-chave: RBCA; NABR; hidrocarbonetos; avaliação de risco; Porto Alegre.

\section{Abstract}

This study defined RBSL (Risk Based Screening Levels) values to hydrocarbons in Porto Alegre, Southern Brazil. The values were obtained through the RBCA methodology, using hydrogeological data from the Crystalline Altered Basement and the Quaternary Sedimentary Deposits. The exposure parameters appropriate to the urban population, as well as toxicity data of selected compounds, were obtained from bibliographical sources and toxicological databases. The reference tables demonstrated that the Crystalline Altered Basement values, in general, are more restrictive than those from the Quaternary Sedimentary Deposits. In comparison to Cetesb's reference tables, the values in Porto Alegre are lower, resulting in the need of more detailed environmental investigations in hydrocarbons polluted areas.

Keywords: RBCA; RBSL; hydrocarbons; risk assessment; Porto Alegre.

\section{Introdução}

A problemática dos passivos ambientais impulsionou o desenvolvimento de estudos científicos e econômicos no sentido de gerenciar melhor as áreas contaminadas, visando a soluções para a proteção humana e ambiental. Vazamentos e derramamentos de derivados do petróleo representam a maioria dos registros de acidentes ambientais no país e no exterior, em virtude do grande número de postos de serviços, distribuidoras, bases e refinarias.
Metodologias de criação de decisões baseadas no risco foram desenvolvidas para o gerenciamento ambiental de áreas impactadas. Nesse contexto, a American Society for Testing and Materials (ASTM) publicou a norma E1739-95, que se refere à metodologia Ação Corretiva Baseada no Risco (RBCA, Risk Based Corrective Action), inicialmente aplicada a hidrocarbonetos e, posteriormente, ampliada para outras substâncias químicas.

A metodologia RBCA é um procedimento tecnicamente defensável, que consiste na tomada de decisões e resposta às 
contaminações baseada no risco a saúde humana. Dessa forma, permite um gerenciamento ambiental mais eficiente, pois prioriza recursos financeiros e técnicos para locais com maior grau de contaminação.

A análise é dividida em três estágios (Tier 1, Tier 2 e Tier 3), que se tornam progressivamente mais detalhados e específicos à medida que progridem para um nível superior. No primeiro estágio de avaliação, Tier 1, são estabelecidos Níveis Aceitáveis Baseados no Risco (NABR), que representam as concentrações máximas aceitáveis no ponto de exposição, calculadas a partir de modelos conceituais conservadores de exposição e parâmetros físicos regionais. Nos Tier 2 e 3 são estabelecidas concentrações-alvo com dados específicos da área de estudo.

Os NABR são resumidos em tabelas de referência, onde valores são apresentados para cada composto de interesse, associados aos cenários de exposição.

No Brasil, estudos de caso são apresentados atendendo análises de problemas ambientais locais, sendo escassas as tabelas de referência em escalas regionais. Neste caso, devem ser ressaltados os dados referentes à cidade de São Paulo, propostos por Maximiano (2001), e os estabelecidos pela Companhia de Tecnologia de Saneamento Ambiental (Cetesb) (2006) para o Estado de São Paulo.

O objetivo do presente estudo foi a elaboração de tabelas de referência de NABR para hidrocarbonetos, com valores calculados a partir das características geológicas e hidrogeológicas do Município de Porto Alegre. Os compostos orgânicos selecionados para esse estudo são benzeno, tolueno, etilbenzeno, xilenos (BTEX), naftaleno e benzo(a)pireno (PAH, Polycyclic Aromatic Hydrocarbons).

\section{Metodologia}

A metodologia para o estabelecimento dos NABR consiste na definição de valores a serem adotados para os dados de entrada que, por sua vez, compreendem parâmetros do meio físico avaliado, parâmetros de exposição e dados toxicológicos e físico-químicos dos compostos orgânicos de interesse.

O caminho de exposição é considerado completo, desde que haja fonte de contaminação, meio físico impactado, mecanismos de transporte e receptor. A identificação desses elementos possibilita estabelecer cenários que representam o modelo conceitual de exposição. A partir desses conceitos e considerando como fonte de contaminação vazamentos de derivados de petróleo, especificamente gasolina e diesel, idealizou-se um único modelo conceitual de exposição para a cidade de Porto Alegre. O modelo aplicado procurou abranger todas as possíveis situações de exposição encontradas no município, admitindo-se receptores adultos com ocupação residencial e comercial.

A caracterização do meio físico foi realizada a partir de dados disponibilizados por empresas privadas e órgãos públicos municipais e federais, e contêm parâmetros como profundidade do nível d'água $\left(\mathrm{L}_{\mathrm{gw}}\right)$, gradiente hidráulico (i), condutividade hidráulica $(K)$, porosidade total $\left(\theta_{t}\right)$, densidade do solo $\left(\rho_{s}\right)$, fração de carbono orgânico (foc) e conteúdo volumétrico de água na zona não saturada $\left(\theta_{\mathrm{ws}}\right)$

Os dados adquiridos foram divididos com base na compartimentação hidrogeológica da região, compreendendo o Embasamento Cristalino alterado e Depósitos Sedimentares Quaternários. Realizou-se um tratamento estatístico dos dados, calculando-se para cada parâmetro as medidas de tendência central, desvio padrão e identificação do maior e menor valor, com o objetivo de registrar um valor único para cada parâmetro no meio de interesse.

Os resultados de NABR foram calculados em planilhas do aplicativo Excel. As equações obedeceram à norma ASTM E1739-95, que não disponibiliza a avaliação de risco por contato dérmico com a água. A equação para esta via de exposição foi elaborada segundo o documento da USEPA (2004B), Risk Assessment Guidance for Superfund, Volume I - Human Health Evaluation Manual (Parte E, Supplemental Guidance for Dermal Risk Assessment).

O procedimento de obtenção da equação NABR para contato dérmico com a água seguiu os passos apresentados pela USEPA (1991), resultando na Equação 1 para compostos carcinogênicos e Equação 2 para compostos não carcinogênicos:

$N A B R_{A B S}=\frac{T R \times B W \times A T_{c} \times 365 \frac{\text { dias }}{\text { ano }}}{2 F A \times K_{p} \sqrt{\frac{6 t_{\text {evento }} \times t_{\text {evento }}}{\pi}} \times E V \times E D \times E F \times S A \times S F_{A B S}}$ Equação 1

$N A B R_{A B S}=\frac{T H Q \times R f D_{A B S} \times B W \times A T_{n} \times 365 \frac{\text { dias }}{\text { ano }}}{2 F A \times K_{p} \sqrt{\frac{6 t_{\text {evento }} \times t_{\text {evento }}}{\pi}} \times E V \times E D \times E F \times S A}$

Equação 2

A lixiviação do solo para a água subterrânea, considerando o contato dérmico com a água no ponto de exposição, foi calculada segundo a Equação 3:

$N A B R_{A B S, L F}=\frac{N A B R_{A B S}}{L F}$ Equação 3

onde:

$\mathrm{T}_{\text {: }}$ tempo de avaliação da exposição para efeitos carcinogênicos (anos);

$\mathrm{AT}_{\mathrm{n}}$ : tempo de avaliação da exposição para efeitos não carcinogênicos (anos);

BW: massa corpórea (kg);

ED: duração da exposição (anos);

EF: freqüência da exposição (dias/ano);

EV: freqüência do evento (eventos/dia);

AS: superfície da pele disponível para contato $\left(\mathrm{cm}^{2}\right)$;

$\mathrm{t}_{\text {evento }}$ : duração do evento (horas/evento); 
$\tau_{\text {evento }}$ : intervalo de tempo por evento (hora/evento), calculado especificamente por composto;

FA: fator de absorção de água (adimensional);

$\mathrm{K}_{\mathrm{p}}$ : coeficiente de permeabilidade dérmica ( $\mathrm{cm} / \mathrm{hora}$ );

$\mathrm{RfD}_{\mathrm{ABS}}$ : dose de referência dérmica;

$\mathrm{SF}_{\mathrm{ABS}}$ : fator de carcinogenicidade dérmica;

THQ: meta de quociente de perigo;

TR: meta de risco carcinogênico;

LF: fator de lixiviação (kg/L), conforme ASTM.

\section{Resultados e discussões}

\section{Dados do meio físico}

\section{Levantamento dos dados}

O levantamento de dados do meio físico resultou em 58 amostras na unidade do Embasamento Cristalino alterado e 66 nos Depósitos Sedimentares Quaternários, totalizando 124 pontos para o Município de Porto Alegre. Deste total, 76 pontos possuem coordenadas geográficas e foram plotados no mapa hidrogeológico (Figura 1), enquanto os demais foram considerados de acordo com as informações geológicas disponíveis nos trabalhos utilizados. Deste último conjunto, 26 amostras (E-1 a E-26) encontram-se no Embasamento Cristalino alterado e 50 (S-1 a S-50) nos Depósitos Sedimentares Quaternários. É possível constatar concentração de pontos na porção Centro-oeste do município em decorrência da maior densidade demográfica dessa região. Já na região Sul, onde há predomínio de atividades agrícolas, são poucos os dados disponíveis.

\section{Tratamento estatístico}

Foram calculadas médias (aritmética, geométrica, harmônica) e mediana, incluindo desvio padrão, para cada parâmetro obtido no levantamento, identificando-se o maior e o menor valor (Tabela 1).

A análise dos dados revela variação pouco significativa entre as duas unidades, o que pode estar relacionado ao limitado número de dados nos Depósitos Sedimentares Quaternários. Nesse caso, embora geologicamente distintas, as amostras da unidade sedimentar da região Norte do município, de origem fluvial, e da região Sul, lacustre, foram reunidas em um único grupo.

Parâmetros adicionais do meio físico

Parâmetros complementares do meio físico foram calculados a partir dos dados levantados (Tabela 2). A taxa de infiltração (I) foi estabelecida com dados de precipitação ( $\mathrm{P}=134,76 \mathrm{~cm} / \mathrm{ano})$ e evapotranspiração $(E T R=91,08 \mathrm{~cm} / \mathrm{ano})$, segundo estudo apresentado pela ESALQ/USP (SENTELHAS et al, 1998).

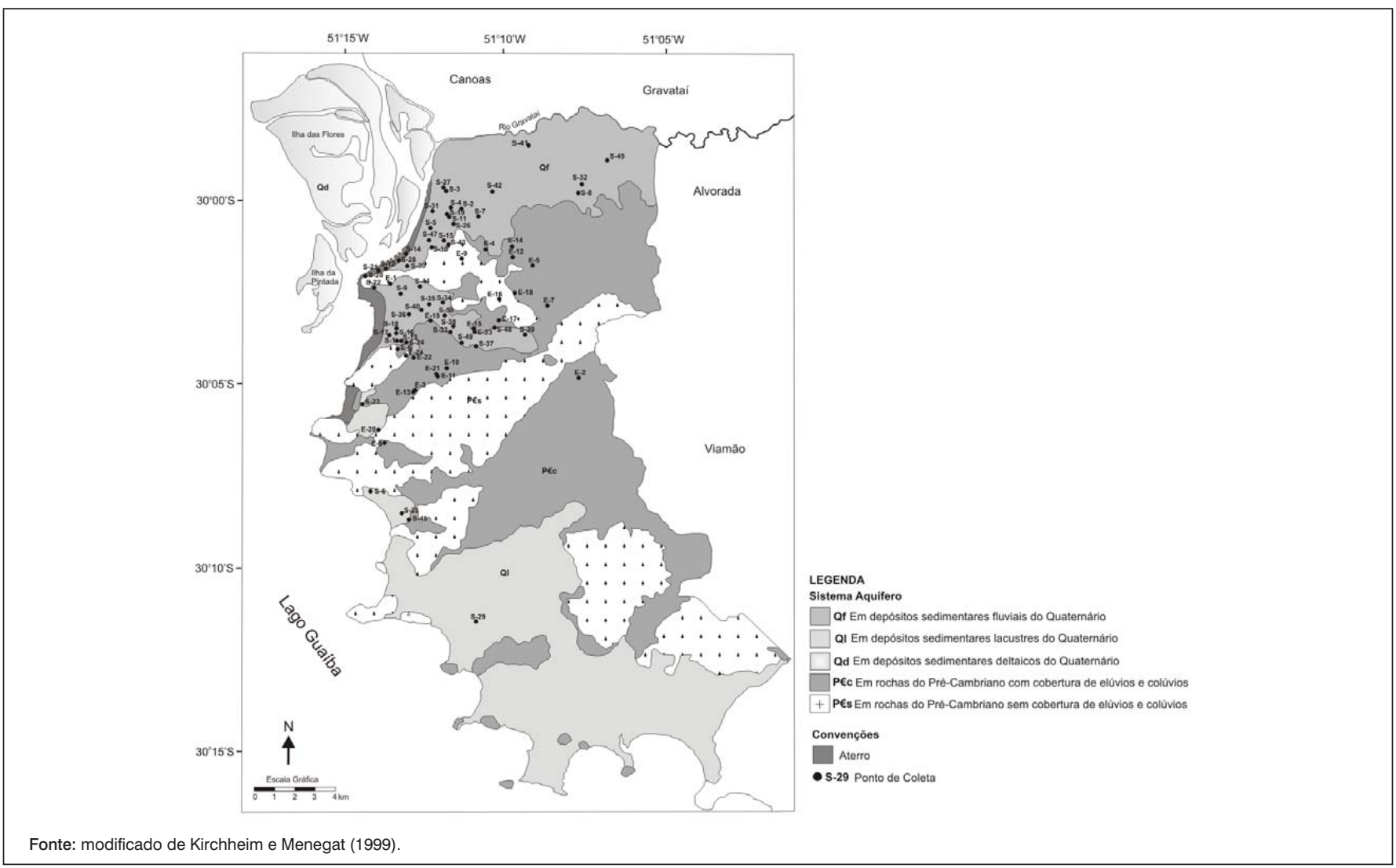

Figura 1 - Mapa hidrogeológico do Município de Porto Alegre com a localização dos pontos de coleta. 
Tabela 1 - Síntese do levantamento de dados para cada parâmetro

\begin{tabular}{|c|c|c|c|c|c|c|c|c|}
\hline \multirow{2}{*}{ Parâmetro } & \multicolumn{4}{|c|}{ Embasamento Cristalino alterado } & \multicolumn{4}{|c|}{ Depósitos Sedimentares Quaternários } \\
\hline & $\mathrm{N}^{\circ}$ dados & Mínimo & Máximo & Valor adotado & $\mathrm{N}^{\circ}$ dados & Mínimo & Máximo & Valor adotado \\
\hline $\mathrm{L}_{\mathrm{gw}}$ - profundidade do nível d'água $(\mathrm{cm})$ & 22 & 47 & 495 & 186 & 48 & 34 & 416 & 129 \\
\hline K - condutividade hidráulica $(\mathrm{cm} / \mathrm{s})$ & 13 & $2,10 \mathrm{E}-6$ & $3,02 \mathrm{E}-4$ & $1,87 \mathrm{E}-5$ & 33 & $8,62 \mathrm{E}-6$ & $2,44 \mathrm{E}-3$ & $3,96 \mathrm{E}-5$ \\
\hline i - gradiente hidráulico & 15 & 0,01 & 0,26 & 0,058 & 43 & 0,003 & 0,24 & 0,02 \\
\hline$\rho_{\mathrm{s}}$ - densidade do solo $\left(\mathrm{g} / \mathrm{cm}^{3}\right)$ & 25 & 1,28 & 1,7 & 1,44 & 5 & 1,42 & 1,7 & 1,59 \\
\hline$\theta_{t}-$ porosidade total & 25 & 0,38 & 0,52 & 0,46 & 4 & 0,38 & 0,6 & 0,46 \\
\hline$\theta_{\text {ws }}$ - conteúdo volumétrico de água & 25 & 0,085 & 0,4 & 0,19 & 9 & 0,103 & 0,583 & 0,308 \\
\hline foc - fração de carbono orgânico & 12 & 0,00013 & 0,0303 & 0,0017 & 22 & 0,0001 & 0,01218 & 0,0006 \\
\hline
\end{tabular}

Tabela 2 - Parâmetros calculados do meio físico em cada unidade hidrogeológica

\begin{tabular}{|c|c|c|c|c|}
\hline Parâmetros calculados & Embasamento cristalino & Depósitos sedimentares & Unidade & Equação \\
\hline $\mathrm{U}_{\mathrm{gw}}-$ velocidade de Darcy & 34,2 & 24,98 & $\mathrm{~cm} / \mathrm{ano}$ & $\mathrm{U}_{\mathrm{gw}}=\mathrm{Kxi}$ \\
\hline$h_{v}-$ espessura da zona não saturada & 181 & 124 & $\mathrm{~cm}$ & $h_{v}=L_{g w}-h_{\text {cap }}$ \\
\hline$\theta_{\text {as }}$ - conteúdo volumétrico de ar na zona não saturada & 0,27 & 0,152 & Adimensional & $\theta_{\mathrm{as}}=\theta_{\mathrm{t}}-\theta_{\mathrm{ws}}$ \\
\hline$\theta_{\text {wcap }}$ - conteúdo volumétrico de água na franja capilar & 0,414 & 0,414 & Adimensional & $\theta_{\text {wcap }}=\theta_{\mathrm{t}} \times 0,90$ \\
\hline$\theta_{\text {acap }}-$ conteúdo volumétrico de ar na franja capilar & 0,046 & 0,046 & Adimensional & $\theta_{\text {acap }}=\theta_{\mathrm{t}} \times 0,10$ \\
\hline I - taxa de infiltração & 43,68 & 43,68 & $\mathrm{~cm} / \mathrm{ano}$ & $\mathrm{I}=\mathrm{P}-\mathrm{ETR}$ \\
\hline
\end{tabular}

Tabela 3 - Valores de propriedades físico-químicas adotados

\begin{tabular}{|c|c|c|c|c|c|c|c|c|}
\hline Propriedades físico-químicas & Benzeno & Tolueno & Etilbenzeno & Xilenos & Naftaleno & Benzo(a)pireno & Unidade & Fonte \\
\hline $\mathrm{H}$ & 0,228 & 0,272 & 0,323 & 0,27 & 1,96E-2 & $4,50 \mathrm{E}-5$ & Adimensional & Usepa (2004C) \\
\hline$D^{\text {air }}$ & $8,80 \mathrm{E}-2$ & $8,70 \mathrm{E}-2$ & $7,50 \mathrm{E}-2$ & $7,14 \mathrm{E}-2$ & $5,90 \mathrm{E}-2$ & $4,30 \mathrm{E}-2$ & $\mathrm{~cm}^{2} / \mathrm{s}$ & Usepa (1996) \\
\hline$D^{\text {wat }}$ & $9,80 \mathrm{E}-6$ & 8,60E-6 & $7,80 \mathrm{E}-6$ & $9,34 \mathrm{E}-6$ & $7,50 \mathrm{E}-6$ & $9,00 \mathrm{E}-6$ & $\mathrm{~cm}^{2} / \mathrm{s}$ & Usepa (1996) \\
\hline $\mathrm{K}_{\mathrm{oc}}$ & 58,9 & 182 & 363 & $443,1^{*}$ & $2,00 E+3$ & $1,02 E+6$ & $\mathrm{~L} / \mathrm{kg}$ & Usepa (1996) \\
\hline MW & 78,1 & 92,4 & 106,2 & 106,2 & 130 & 250 & $\mathrm{~g} / \mathrm{mol}$ & Usepa (2004C) \\
\hline S & 1750 & 526 & 169 & 110 & 31 & $1,62 \mathrm{E}-3$ & $\mathrm{mg} / \mathrm{L}$ & Usepa (2004C) \\
\hline$P_{v}$ & 95,2 & 30 & 9,51 & 8 & $8,50 \mathrm{E}-2$ & $5,50 \mathrm{E}-9$ & $\mathrm{mmHg}$ & Usepa (2004C) \\
\hline
\end{tabular}

H: Coeficiente da Lei de Henry; Dair: Coeficiente de difusão no ar; Dwat: Coeficiente de difusão na água; MW: Peso molecular; S: Solubilidade; $\mathrm{P}_{\mathrm{v}}$ : Pressão de vapor; $\mathrm{K}_{\mathrm{oc}}$ : Coeficiente de partição carbono orgânico-água. *Para xilenos, adotaram-se valores do RAIS (2007).

Os demais parâmetros necessários ao cálculo dos NABR foram adotados conforme os valores propostos pela ASTM (1995), com exceção da medida de área-fonte em direção paralela ao fluxo subterrâneo e ao vento (W), para a qual se atribuiu valor de $4500 \mathrm{~cm}$ (CETESB, 2006).

\section{Propriedades físico-químicas dos compostos orgânicos}

Os valores referentes às propriedades físico-químicas dos compostos orgânicos de interesse estão listados na Tabela 3.

O coeficiente da Lei de Henry $(\mathrm{H})$ foi definido com valores de constante de Henry $\left(\mathrm{K}_{\mathrm{H}}\right.$ ) obtidos do banco SCDM da USEPA (2004C) para os compostos selecionados e temperatura de $298 \mathrm{~K}$.

\section{Dados de toxicidade}

Valores de toxicidade são apresentados na Tabela 4, na qual dados relacionados à inalação $\left(\mathrm{SF}_{\mathrm{i}}\right.$ e $\left.\mathrm{RfD}_{\mathrm{i}}\right)$ e absorção dérmicas $\left(\mathrm{SF}_{\mathrm{ABS}} \mathrm{e}\right.$ $\mathrm{RfD}_{\mathrm{ABS}}$ ) foram calculados conforme critérios de ajustes estabelecidos pelos documentos PRG - Region 9 (USEPA, 2004A) e RAGS -Part E, Supplemental Guidance for Dermal Risk Assessment (USEPA, 2004B), respectivamente.

O risco carcinogênico admitido para esse estudo é de $10^{-5}$. Este valor indica que um indivíduo exposto a uma determinada substância química tem uma chance adicional em 100 mil de desenvolver câncer durante a vida. A caracterização do risco para compostos não carcinogênicos é expressa pelo quociente de perigo, que é dada pela razão entre a dose de ingresso específica para cada composto e a dose 
Tabela 4 - Dados de toxicidade de compostos selecionados

\begin{tabular}{|c|c|c|c|c|c|c|c|c|}
\hline Parâmetros & Benzeno & Tolueno & Etilbenzeno & Xilenos & Naftaleno & Benzo(a)pireno & Unidade & Fonte \\
\hline $\mathrm{SF}_{\circ}$ & $5,50 \mathrm{E}-2$ & - & - & - & - & 7,3 & (mg/kg-dia) $)^{-1}$ & IRIS (2007) \\
\hline $\mathrm{SF}_{\mathrm{i}}$ & 2,73E-2 & - & - & - & - & 3,08 & (mg/kg-dia) $)^{-1}$ & Calculado \\
\hline $\mathrm{SF}_{\mathrm{ABS}}$ & $5,67 \mathrm{E}-2$ & - & - & - & - & 23,55 & $(\mathrm{mg} / \mathrm{kg}-\mathrm{dia})^{-1}$ & Calculado \\
\hline $\mathrm{RfD}_{\text {。 }}$ & $4,00 \mathrm{E}-3$ & $8,00 \mathrm{E}-2$ & $1,00 \mathrm{E}-1$ & $2,00 \mathrm{E}-1$ & $2,00 \mathrm{E}-2$ & - & $\mathrm{mg} / \mathrm{kg}$-dia & IRIS (2007) \\
\hline $\operatorname{RfD}_{i}$ & 8,57E-3 & $1,42 E+0$ & $2,86 \mathrm{E}-1$ & $2,86 \mathrm{E}-2$ & $8,57 \mathrm{E}-4$ & - & $\mathrm{mg} / \mathrm{kg}$-dia & Calculado \\
\hline $\mathrm{RfD}_{\mathrm{ABS}}$ & $3,88 \mathrm{E}-3$ & $6,40 \mathrm{E}-2$ & $9,70 \mathrm{E}-2$ & $1,84 \mathrm{E}-1$ & $1,60 \mathrm{E}-2$ & - & $\mathrm{mg} / \mathrm{kg}$-dia & Calculado \\
\hline $\mathrm{RAF}_{\text {。 }}$ & 1 & 1 & 1 & 1 & 1 & 1 & Adimensional & ASTM (1995) \\
\hline $\operatorname{RAF}_{\mathrm{d}}$ & 0,5 & 0,5 & 0,5 & 0,5 & 0,05 & 0,05 & Adimensional & ASTM (1995) \\
\hline $\mathrm{K}_{\mathrm{p}}$ & $1,50 \mathrm{E}-2$ & $3,10 \mathrm{E}-2$ & 4,90E-2 & $5,30 \mathrm{E}-2$ & $4,70 \mathrm{E}-2$ & $7,00 \mathrm{E}-1$ & $\mathrm{~cm} /$ hora & Usepa (2004B) \\
\hline FA & 1 & 1 & 1 & 1 & 1 & 1 & Adimensional & Usepa (2004B) \\
\hline $\mathrm{ABS}_{\mathrm{Gl}}$ & 0,97 & 0,8 & 0,97 & 0,92 & 0,8 & 0,31 & Adimensional & RAIS (2007) \\
\hline $\operatorname{RfC}_{i}$ & $3,00 \mathrm{E}-2$ & $5,00 \mathrm{E}+0$ & $1,00 \mathrm{E}+0$ & $1,00 \mathrm{E}-1$ & $3,00 \mathrm{E}-3$ & - & $\mathrm{mg} / \mathrm{m}^{3}$ & IRIS (2007) \\
\hline URF $_{i}$ & $7,80 \mathrm{E}-3$ & - & - & - & - & $8,80 \mathrm{E}-1$ * & $\left(\mathrm{mg} / \mathrm{m}^{3}\right)^{-1}$ & IRIS (2007) \\
\hline
\end{tabular}

$\mathrm{SF}_{0}$ : Fator de carcinogenicidade oral; $\mathrm{SF}_{\mathrm{i}}$ : Fator de carcinogenicidade para inalação; $\mathrm{SF}_{\mathrm{ABS}}$ : Fator de carcinogenicidade dérmica; $\mathrm{RfD}_{0}$ : Dose de referência oral; $\mathrm{RfD}_{\mathrm{i}}$ : Dose de referência para inalação; $\mathrm{RfD}_{\mathrm{AB}}$ : Dose de referência dérmica; $\mathrm{RAF}$ : Fator de absorção relativa oral; $\mathrm{RAF}_{\mathrm{d}}$ : Fator de absorção dérmica relativa; $\mathrm{FA}$ : Fração absorvida de água; $\mathrm{K}_{\mathrm{p}}$ : Coeficiente de permeabilidade dérmica. $\mathrm{ABS}_{\mathrm{Gl}}$ : Fator de absorção gastrintestinal; RfC: Concentração de referência para inalação; URF; : Fator unidade de risco; *Para benzo(a)pireno adotou-se valor do RAIS (2007).

Tabela 5 - Parâmetros de exposição utilizados nos cenários do Município de Porto Alegre

\begin{tabular}{|c|c|c|c|c|}
\hline Parâmetros de Exposição & Residenciais & Comerciais & Unidade & Fonte \\
\hline BW - massa corpórea & 60 & 60 & $\mathrm{~kg}$ & Cetesb (2001) \\
\hline$A T_{c}$ - tempo de avaliação da exposição para efeitos carcinogênicos & 68 & 68 & anos & Cetesb (2006) \\
\hline $\mathrm{AT}_{\mathrm{n}}$ - tempo de avaliação da exposição para efeitos não carcinogênicos & 45 & 45 & anos & Cetesb (2006) \\
\hline EF - frequência da exposição & 350 & 270 & dias/ano & Cetesb (2006) \\
\hline ED - duração da exposição & 45 & 45 & anos & Cetesb (2006) \\
\hline $\mathrm{IR}_{\mathrm{ar}}$ - taxa de inalação do ar (ambiente interno) & 22 & 22 & $\mathrm{~m}^{3} / \mathrm{dia}$ & Cetesb (2001) \\
\hline $\mathrm{IR}_{\mathrm{ar}}$ - taxa de inalação do ar (ambiente externo) & 22 & 22 & $\mathrm{~m}^{3} / \mathrm{dia}$ & Cetesb (2001) \\
\hline $\mathrm{IR}_{\mathrm{w}}$ - taxa de ingestão de água subterrânea & 2 & 1 & L/dia & Cetesb (2001) \\
\hline $\mathrm{IR}_{\mathrm{s}}$ - taxa de ingestão de solo & 100 & 50 & $\mathrm{mg} / \mathrm{dia}$ & Cetesb (2001) \\
\hline $\mathrm{SA}_{\mathrm{s}}$ - área superficial da pele disponível para contato com o solo & 8.600 & 2.000 & $\mathrm{~cm}^{2}$ & Cetesb (2001) \\
\hline $\mathrm{SA}_{\mathrm{w}}$ - área superficial da pele disponível para contato com a água & 16.600 & 16.600 & $\mathrm{~cm}^{2}$ & Cetesb (2001) \\
\hline AF - fator de aderência do solo na pele & 0,5 & 0,5 & $\mathrm{mg} / \mathrm{cm}^{2}$ & ASTM (1995) \\
\hline EV - frequência do evento & 1 & 1 & evento/dia & Usepa (2004B) \\
\hline$t_{\text {evento }}$ - duração do evento & 0,58 & 0,58 & h/evento & Usepa (2004B) \\
\hline
\end{tabular}

Nota: O termo 'evento', citado nos parâmetros EV e tevento, refere-se ao banho por ducha ou imersão.

de referência correspondente. Assim, quando o quociente de perigo for superior a 1, há possibilidade maior de ocorrência de efeitos tóxicos à saúde humana.

\section{Parâmetros de exposição}

A elaboração da lista de parâmetros de exposição para cenários aplicados ao Município de Porto Alegre levou em consideração os valores adotados pela Cetesb (Tabela 5). No caso de ausência de informação neste órgão, assumiram-se valores referenciados pela ASTM e USEPA

\section{Resultados dos NABR para o município de Porto Alegre}

As Tabelas 6, 7 e 8 apresentam resultados de NABR para os compostos benzeno, tolueno, etilbenzeno, xilenos, naftaleno e benzo(a) pireno nas duas unidades hidrogeológicas consideradas. Os valores foram comparados aos limites físico-químicos da fase do composto no meio de interesse (solubilidade, concentração limite de saturação de fase retida no solo e concentração limite de saturação de vapores) para todos os caminhos de exposição elaborados no modelo conceitual. Dessa forma, os valores destacados com asterisco ultrapassaram 
Tabela 6 - Tabela de referência dos compostos benzeno e benzo(a)pireno para as unidades Embasamento Cristalino alterado e Depósitos Sedimentares Quaternários

\begin{tabular}{|c|c|c|c|c|c|c|c|c|c|c|}
\hline \multirow{3}{*}{ Cenários } & \multirow{3}{*}{ Unidade } & \multirow{3}{*}{ Risco } & \multicolumn{4}{|c|}{ Benzeno - CAS RN 71-43-2 } & \multicolumn{4}{|c|}{ Benzeno(a)pireno - CAS RN 50-32-8 } \\
\hline & & & \multicolumn{2}{|c|}{$\begin{array}{c}\text { NABR - Embasamento } \\
\text { Cristalino }\end{array}$} & \multicolumn{2}{|c|}{$\begin{array}{l}\text { NABR - Depósitos } \\
\text { Sedimentares }\end{array}$} & \multicolumn{2}{|c|}{$\begin{array}{c}\text { NABR - Embasamento } \\
\text { Cristalino }\end{array}$} & \multicolumn{2}{|c|}{$\begin{array}{l}\text { NABR - Depósitos } \\
\text { Sedimentares }\end{array}$} \\
\hline & & & Residencial & Comercial & Residencial & Comercial & Residencial & Comercial & Residencial & Comercial \\
\hline $\begin{array}{l}\text { Inalação de vapores } \\
\text { em ambientes } \\
\text { abertos }\end{array}$ & $\mu \mathrm{g} / \mathrm{m}^{3}$ & 1,00E-5 & $1,57 \mathrm{E}+0$ & $2,04 \mathrm{E}+0$ & $1,57 \mathrm{E}+0$ & $2,04 \mathrm{E}+0$ & $1,40 \mathrm{E}-2^{*}$ & $1,81 \mathrm{E}-2 *$ & $1,40 \mathrm{E}-2^{*}$ & $1,81 \mathrm{E}-2^{*}$ \\
\hline $\begin{array}{l}\text { Inalação de vapores } \\
\text { em ambientes } \\
\text { fechados }\end{array}$ & $\mu \mathrm{g} / \mathrm{m}^{3}$ & 1,00E-5 & $1,57 E+0$ & $2,04 \mathrm{E}+0$ & $1,57 \mathrm{E}+0$ & $2,04 \mathrm{E}+0$ & $1,40 \mathrm{E}-2 *$ & $1,81 \mathrm{E}-2 *$ & $1,40 \mathrm{E}-2^{*}$ & $1,81 \mathrm{E}-2^{*}$ \\
\hline $\begin{array}{l}\text { Ingestão de água } \\
\text { subterrânea }\end{array}$ & $\mathrm{mg} / \mathrm{L}$ & $1,00 \mathrm{E}-5$ & $8,60 \mathrm{E}-3$ & $2,23 \mathrm{E}-2$ & $8,60 \mathrm{E}-3$ & 2,23E-2 & $6,48 \mathrm{E}-5$ & $1,68 \mathrm{E}-4$ & $6,48 \mathrm{E}-5$ & $1,68 \mathrm{E}-4$ \\
\hline $\begin{array}{l}\text { Contato dérmico } \\
\text { com a água subter- } \\
\text { rânea }\end{array}$ & $\mathrm{mg} / \mathrm{L}$ & 1,00E-5 & 5,93E-2 & 7,69E-2 & 5,93E-2 & $7,69 \mathrm{E}-2$ & $1,01 \mathrm{E}-6$ & $1,31 \mathrm{E}-6$ & $1,01 \mathrm{E}-6$ & 1,31E-6 \\
\hline $\begin{array}{l}\text { Ingestão, contato } \\
\text { dérmico e inalação } \\
\text { de vapores e } \\
\text { partículas a partir } \\
\text { do solo superficial }\end{array}$ & $\mathrm{mg} / \mathrm{kg}$ & 1,00E-5 & $7,02 \mathrm{E}+0$ & $2,97 \mathrm{E}+1$ & $6,96 \mathrm{E}+0$ & $2,89 \mathrm{E}+1$ & $4,10 \mathrm{E}-1$ * & $1,67 \mathrm{E}+0$ * & $4,09 \mathrm{E}-1$ * & $1,65 \mathrm{E}+0$ * \\
\hline $\begin{array}{l}\text { Inalação de vapores } \\
\text { em ambientes aber- } \\
\text { tos a partir do solo } \\
\text { subsuperficial }\end{array}$ & $\mathrm{mg} / \mathrm{kg}$ & $1,00 \mathrm{E}-5$ & $3,57 \mathrm{E}-1$ & $4,63 \mathrm{E}-1$ & $2,20 \mathrm{E}+0$ & $2,85 \mathrm{E}+0$ & $8,48 \mathrm{E}+4^{*}$ & $1,10 \mathrm{E}+5^{\star}$ & $9,94 \mathrm{E}+3^{*}$ & $1,29 \mathrm{E}+4^{*}$ \\
\hline $\begin{array}{l}\text { Inalação de vapores } \\
\text { em ambientes } \\
\text { fechados a partir do } \\
\text { solo subsuperficial }\end{array}$ & $\mathrm{mg} / \mathrm{kg}$ & $1,00 \mathrm{E}-5$ & $1,80 \mathrm{E}-2$ & $5,75 \mathrm{E}-2$ & 2,17E-2 & $6,92 \mathrm{E}-2$ & $7,52 \mathrm{E}+3^{*}$ & $2,40 \mathrm{E}+4^{\star}$ & $2,60 \mathrm{E}+3^{\star}$ & $8,30 \mathrm{E}+3^{*}$ \\
\hline $\begin{array}{l}\text { Inalação de vapores } \\
\text { em ambientes aber- } \\
\text { tos a partir da água } \\
\text { subterrânea }\end{array}$ & $\mathrm{mg} / \mathrm{L}$ & $1,00 \mathrm{E}-5$ & $1,59 \mathrm{E}+1$ & $2,06 \mathrm{E}+1$ & $2,44 \mathrm{E}+1$ & $3,17 \mathrm{E}+1$ & $8,88 \mathrm{E}+1^{*}$ & $1,15 \mathrm{E}+2^{\star}$ & $2,04 \mathrm{E}+1$ * & $2,65 \mathrm{E}+1^{*}$ \\
\hline $\begin{array}{l}\text { Inalação de vapores } \\
\text { em ambientes } \\
\text { fechados a partir da } \\
\text { água subterrânea }\end{array}$ & $\mathrm{mg} / \mathrm{L}$ & $1,00 \mathrm{E}-5$ & $1,07 \mathrm{E}-1$ & $3,40 \mathrm{E}-1$ & $1,30 \mathrm{E}-1$ & $4,16 \mathrm{E}-1$ & $4,45 \mathrm{E}+0$ * & $1,42 \mathrm{E}+1$ * & $4,26 \mathrm{E}+0$ * & $1,36 \mathrm{E}+1$ * \\
\hline $\begin{array}{l}\text { Ingestão de água } \\
\text { subterrânea a partir } \\
\text { da lixiviação do solo } \\
\text { subsuperficial }\end{array}$ & $\mathrm{mg} / \mathrm{kg}$ & $1,00 \mathrm{E}-5$ & $2,44 \mathrm{E}-3$ & $6,34 \mathrm{E}-3$ & $2,21 \mathrm{E}-3$ & $5,73 E-3$ & $1,16 \mathrm{E}-1$ * & $3,01 \mathrm{E}-1$ * & $4,07 \mathrm{E}-2^{\star}$ & $1,05 \mathrm{E}-1^{*}$ \\
\hline $\begin{array}{l}\text { Contato dérmico } \\
\text { com a água subter- } \\
\text { rânea a partir da } \\
\text { lixiviação do solo } \\
\text { subsuperficial }\end{array}$ & $\mathrm{mg} / \mathrm{kg}$ & 1,00E-5 & 1,69E-2 & $2,19 \mathrm{E}-2$ & $1,53 \mathrm{E}-2$ & $1,98 \mathrm{E}-2$ & $1,81 \mathrm{E}-3 *$ & $2,35 \mathrm{E}-3^{*}$ & $6,34 \mathrm{E}-4^{\star}$ & $8,22 \mathrm{E}-4^{*}$ \\
\hline
\end{tabular}

*Valores que ultrapassaram os limites físico-químicos de ocorrência do composto no meio avaliado e não devem ser utilizados na avaliação de risco.

os limites correspondentes aos cenários de exposição e, portanto, não devem ser utilizados na avaliação de risco.

Os caminhos de exposição direta (inalação de vapores em ambientes fechados e abertos, ingestão de água subterrânea e contato dérmico com a água) resultaram em valores iguais para as duas unidades. Esse fato é resultado das equações de NABR considerarem somente parâmetros de exposição e toxicidade dos compostos para cenários diretos

Os NABR para a inalação de vapores em ambientes abertos e fechados foram iguais devido à utilização do mesmo valor para parâmetros de taxa de inalação do ar em ambientes fechado e aberto.
Conforme se observou nas tabelas de referências, de maneira geral, verifica-se que os NABR para Embasamento Cristalino alterado são mais restritivos do que os obtidos para Depósitos Sedimentares Quaternários

Os cenários de inalação de vapores provenientes do solo e água subterrânea para os compostos BTEX apresentaram-se menos conservadores na unidade sedimentar, com exceção do caminho de inalação de vapores do solo em ambientes fechados. O valor de porosidade total $\left(\theta_{\mathrm{t}}\right)$ utilizado foi 0,46 para as duas unidades, e o conteúdo volumétrico de ar na zona não saturada $\left(\theta_{\mathrm{as}}\right)$ é menor nos Depósitos Sedimentares $(0,152)$ do que no Embasamento Cristalino $(0,27)$. 
Tabela 7 - Tabela de referência dos compostos tolueno e etilbenzeno para as unidades Embasamento Cristalino alterado e Depósitos Sedimentares Quaternários

\begin{tabular}{|c|c|c|c|c|c|c|c|c|c|c|}
\hline \multirow{3}{*}{ Cenários } & \multirow{3}{*}{ Unidade } & \multirow{3}{*}{ Risco } & \multicolumn{4}{|c|}{ Tolueno - CAS RN 108-88-3 } & \multicolumn{4}{|c|}{ Etilbenzeno - CAS RN 100-41-4 } \\
\hline & & & \multicolumn{2}{|c|}{$\begin{array}{c}\text { NABR - Embasamento } \\
\text { Cristalino }\end{array}$} & \multicolumn{2}{|c|}{$\begin{array}{l}\text { NABR - Depósitos } \\
\text { Sedimentares }\end{array}$} & \multicolumn{2}{|c|}{$\begin{array}{c}\text { NABR - Embasamento } \\
\text { Cristalino }\end{array}$} & \multicolumn{2}{|c|}{$\begin{array}{l}\text { NABR - Depósitos } \\
\text { Sedimentares }\end{array}$} \\
\hline & & & Residencial & Comercial & Residencial & Comercial & Residencial & Comercial & Residencial & Comercial \\
\hline $\begin{array}{l}\text { Inalação de vapores } \\
\text { em ambientes } \\
\text { abertos }\end{array}$ & $\mu \mathrm{g} / \mathrm{m}^{3}$ & 1 & $4,04 \mathrm{E}+3$ & $5,24 \mathrm{E}+3$ & $4,04 \mathrm{E}+3$ & $5,24 \mathrm{E}+3$ & $8,13 E+2$ & $1,05 E+3$ & $8,13 E+2$ & $1,05 E+3$ \\
\hline $\begin{array}{l}\text { Inalação de vapores } \\
\text { em ambientes } \\
\text { fechados }\end{array}$ & $\mu \mathrm{g} / \mathrm{m}^{3}$ & 1 & $4,04 \mathrm{E}+3$ & $5,24 \mathrm{E}+3$ & $4,04 E+3$ & $5,24 \mathrm{E}+3$ & $8,13 E+2$ & $1,05 E+3$ & $8,13 E+2$ & $1,05 E+3$ \\
\hline $\begin{array}{l}\text { Ingestão de água } \\
\text { subterrânea }\end{array}$ & $\mathrm{mg} / \mathrm{L}$ & 1 & $2,50 \mathrm{E}+0$ & $6,49 \mathrm{E}+0$ & $2,50 \mathrm{E}+0$ & $6,49 \mathrm{E}+0$ & $3,13 E+0$ & $8,11 \mathrm{E}+0$ & $3,13 E+0$ & $8,11 \mathrm{E}+0$ \\
\hline $\begin{array}{l}\text { Contato dérmico } \\
\text { com a água subter- } \\
\text { rânea }\end{array}$ & $\mathrm{mg} / \mathrm{L}$ & 1 & $6,29 E+0$ & $8,15 E+0$ & $6,29 E+0$ & $8,15 E+0$ & $5,52 E+0$ & $7,15 E+0$ & $5,52 \mathrm{E}+0$ & $7,15 E+0$ \\
\hline $\begin{array}{l}\text { Ingestão, contato } \\
\text { dérmico e inalação } \\
\text { de vapores e } \\
\text { partículas a partir } \\
\text { do solo superficial }\end{array}$ & $\mathrm{mg} / \mathrm{kg}$ & 1 & $2,20 \mathrm{E}+3^{*}$ & $1,13 \mathrm{E}+4^{*}$ & $2,20 \mathrm{E}+3^{*}$ & $1,13 \mathrm{E}+4^{*}$ & $2,62 \mathrm{E}+3^{\star}$ & $1,17 \mathrm{E}+4^{*}$ & $2,60 \mathrm{E}+3^{*}$ & $1,15 \mathrm{E}+4^{*}$ \\
\hline $\begin{array}{l}\text { Inalação de vapores } \\
\text { em ambientes aber- } \\
\text { tos a partir do solo } \\
\text { subsuperficial }\end{array}$ & $\mathrm{mg} / \mathrm{kg}$ & 1 & $1,39 \mathrm{E}+3^{*}$ & $1,80 \mathrm{E}+3^{*}$ & $6,27 \mathrm{E}+3^{*}$ & $8,13 E+3^{*}$ & $4,50 \mathrm{E}+2^{*}$ & $5,84 \mathrm{E}+2^{\star}$ & $1,66 \mathrm{E}+3^{*}$ & $2,15 \mathrm{E}+3^{*}$ \\
\hline $\begin{array}{l}\text { Inalação de vapores } \\
\text { em ambientes } \\
\text { fechados a partir do } \\
\text { solo subsuperficial }\end{array}$ & $\mathrm{mg} / \mathrm{kg}$ & 1 & $7,02 E+1$ & $2,24 \mathrm{E}+2$ & $6,18 E+1$ & $1,98 \mathrm{E}+2$ & $2,27 E+1$ & $7,25 E+1$ & $1,64 \mathrm{E}+1$ & $5,23 E+1$ \\
\hline $\begin{array}{l}\text { Inalação de vapores } \\
\text { em ambientes aber- } \\
\text { tos a partir da água } \\
\text { subterrânea }\end{array}$ & $\mathrm{mg} / \mathrm{L}$ & 1 & $3,82 \mathrm{E}+4^{*}$ & $4,96 \mathrm{E}+4^{\star}$ & $5,68 \mathrm{E}+4^{*}$ & $7,36 \mathrm{E}+4^{*}$ & $7,80 \mathrm{E}+3^{*}$ & $1,01 \mathrm{E}+4^{*}$ & $1,14 \mathrm{E}+4^{*}$ & $1,48 \mathrm{E}+4^{*}$ \\
\hline $\begin{array}{l}\text { Inalação de vapores } \\
\text { em ambientes } \\
\text { fechados a partir da } \\
\text { água subterrânea }\end{array}$ & $\mathrm{mg} / \mathrm{L}$ & 1 & $2,42 E+2$ & $7,72 \mathrm{E}+2^{\star}$ & $2,94 \mathrm{E}+2$ & $9,38 \mathrm{E}+2^{*}$ & $4,83 E+1$ & $1,54 \mathrm{E}+2$ & $5,85 E+1$ & $1,87 \mathrm{E}+2^{*}$ \\
\hline $\begin{array}{l}\text { Ingestão de água } \\
\text { subterrânea a partir } \\
\text { da lixiviação do solo } \\
\text { subsuperficial }\end{array}$ & $\mathrm{mg} / \mathrm{kg}$ & 1 & $1,28 E+0$ & $3,31 E+0$ & $8,44 \mathrm{E}-1$ & $2,19 \mathrm{E}+0$ & $2,62 E+0$ & $6,80 \mathrm{E}+0$ & $1,42 E+0$ & $3,68 E+0$ \\
\hline $\begin{array}{l}\text { Contato dérmico } \\
\text { com a água subter- } \\
\text { rânea a partir da } \\
\text { lixiviação do solo } \\
\text { subsuperficial }\end{array}$ & $\mathrm{mg} / \mathrm{kg}$ & 1 & $3,20 E+0$ & $4,15 E+0$ & $2,12 \mathrm{E}+0$ & $2,75 E+0$ & $4,62 E+0$ & $5,99 E+0$ & $2,50 E+0$ & $3,24 \mathrm{E}+0$ \\
\hline
\end{tabular}

*Valores que ultrapassaram os limites físico-químicos de ocorrência do composto no meio avaliado e não devem ser utilizados na avaliação de risco.

Assim, os NABR são maiores no compartimento sedimentar no qual é mais reduzido o volume de poros com ar para volatilização.

Em contrapartida, os NABR para caminhos de ingestão e contato dérmico com água subterrânea, a partir da lixiviação de solo, foram mais restritivos nos Depósitos Sedimentares em razão do maior fator de lixiviação (LF). Com base na equação do LF proposta pela ASTM, o maior valor na unidade sedimentar se relaciona à menor velocidade de Darcy $\left(\mathrm{U}_{\mathrm{gw}}\right)$ e ao maior coeficiente de partição fase retida/água intersticial do solo $\left(\mathrm{K}_{\mathrm{sw}}\right)$.

Os valores obtidos para o cenário de ingestão, contato dérmico e inalação de vapores e partículas a partir do solo superficial são próximos para as duas unidades. Esta constatação é feita em razão da proximidade dos fatores de volatilização do solo superficial com a zona de respiração $\left(\mathrm{VF}_{\text {ssamb }}\right)$ dos dois meios avaliados, já que o fator de emissão de partículas (PEF) é igual para ambas as unidades. Dessa forma, o único parâmetro que interfere na equação de NABR para esse caminho de exposição é o $\mathrm{VF}_{\text {ssamb }}$, que pouco influencia o resultado.

Os NABR obtidos para os compostos naftaleno e benzo(a)pireno, em sua maioria, foram superiores aos limites de ocorrência do composto no meio avaliado. Assim, os valores excedidos não devem ser utilizados como referência em avaliações de risco. Esta limitação relaciona-se aos elevados coeficientes de partição carbono orgânico/ 
Tabela 8 - Tabela de referência dos compostos xilenos e naftaleno para as unidades Embasamento Cristalino alterado e Depósitos Sedimentares Quaternários

\begin{tabular}{|c|c|c|c|c|c|c|c|c|c|c|}
\hline \multirow{3}{*}{ Cenários } & \multirow{3}{*}{ Unidade } & \multirow{3}{*}{ Risco } & \multicolumn{4}{|c|}{ Xilenos totais - CAS RN 1330-20-7 } & \multicolumn{4}{|c|}{ Naftaleno - CAS RN 91-20-3 } \\
\hline & & & \multicolumn{2}{|c|}{$\begin{array}{c}\text { NABR - Embasamento } \\
\text { Cristalino }\end{array}$} & \multicolumn{2}{|c|}{$\begin{array}{l}\text { NABR - Depósitos } \\
\text { Sedimentares }\end{array}$} & \multicolumn{2}{|c|}{$\begin{array}{c}\text { NABR - Embasamento } \\
\text { Cristalino }\end{array}$} & \multicolumn{2}{|c|}{$\begin{array}{l}\text { NABR - Depósitos } \\
\text { Sedimentares }\end{array}$} \\
\hline & & & Residencial & Comercial & Residencial & Comercial & Residencial & Comercial & Residencial & Comercial \\
\hline $\begin{array}{l}\text { Inalação de vapores } \\
\text { em ambientes } \\
\text { abertos }\end{array}$ & $\mu \mathrm{g} / \mathrm{m}^{3}$ & 1 & $8,13 E+1$ & $1,05 E+2$ & $8,13 E+1$ & $1,05 \mathrm{E}+2$ & $2,44 \mathrm{E}+0$ & $3,16 \mathrm{E}+0$ & $2,44 \mathrm{E}+0$ & $3,16 \mathrm{E}+0$ \\
\hline $\begin{array}{l}\text { Inalação de vapores } \\
\text { em ambientes } \\
\text { fechados }\end{array}$ & $\mu \mathrm{g} / \mathrm{m}^{3}$ & 1 & $8,13 E+1$ & $1,05 E+2$ & $8,13 E+1$ & $1,05 \mathrm{E}+2$ & $2,44 \mathrm{E}+0$ & $3,16 \mathrm{E}+0$ & $2,44 \mathrm{E}+0$ & $3,16 \mathrm{E}+0$ \\
\hline $\begin{array}{l}\text { Ingestão de água } \\
\text { subterrânea }\end{array}$ & $\mathrm{mg} / \mathrm{L}$ & 1 & $6,26 \mathrm{E}+0$ & $1,62 \mathrm{E}+1$ & $6,26 \mathrm{E}+0$ & $1,62 E+1$ & $6,26 \mathrm{E}-1$ & $1,62 \mathrm{E}+0$ & $6,26 \mathrm{E}-1$ & $1,62 E+0$ \\
\hline $\begin{array}{l}\text { Contato dérmico } \\
\text { com a água subter- } \\
\text { rânea }\end{array}$ & $\mathrm{mg} / \mathrm{L}$ & 1 & $9,67 E+0$ & $1,25 E+1$ & $9,67 E+0$ & $1,25 E+1$ & $8,14 \mathrm{E}-1$ & $1,05 E+0$ & $8,14 \mathrm{E}-1$ & $1,05 E+0$ \\
\hline $\begin{array}{l}\text { Ingestão, contato } \\
\text { dérmico e inalação } \\
\text { de vapores e } \\
\text { partículas a partir } \\
\text { do solo superficial }\end{array}$ & $\mathrm{mg} / \mathrm{kg}$ & 1 & $2,47 \mathrm{E}+3^{*}$ & $4,83 E+3 *$ & $2,34 \mathrm{E}+3^{\star}$ & $4,44 \mathrm{E}+3^{\star}$ & $1,29 E+2 *$ & $1,71 \mathrm{E}+2^{\star}$ & $1,64 \mathrm{E}+2^{\star}$ & $2,19 \mathrm{E}+2^{\star}$ \\
\hline $\begin{array}{l}\text { Inalação de vapores } \\
\text { em ambientes aber- } \\
\text { tos a partir do solo } \\
\text { subsuperficial }\end{array}$ & $\mathrm{mg} / \mathrm{kg}$ & 1 & $6,54 \mathrm{E}+1$ & $8,48 E+1$ & $2,29 E+2^{*}$ & $2,96 \mathrm{E}+2^{*}$ & $1,23 E+2^{*}$ & $1,60 \mathrm{E}+2^{\star}$ & $3,09 \mathrm{E}+2^{*}$ & $4,01 E+2^{*}$ \\
\hline $\begin{array}{l}\text { Inalação de vapores } \\
\text { em ambientes } \\
\text { fechados a partir do } \\
\text { solo subsuperficial }\end{array}$ & $\mathrm{mg} / \mathrm{kg}$ & 1 & $3,30 E+0$ & $1,05 E+1$ & $2,26 \mathrm{E}+0$ & $7,20 \mathrm{E}+0$ & $6,22 \mathrm{E}+0$ & $1,99 \mathrm{E}+1^{*}$ & $3,18 \mathrm{E}+0$ & $1,02 E+1$ \\
\hline $\begin{array}{l}\text { Inalação de vapores } \\
\text { em ambientes aber- } \\
\text { tos a partir da água } \\
\text { subterrânea }\end{array}$ & $\mathrm{mg} / \mathrm{L}$ & 1 & $8,59 \mathrm{E}+2^{\star}$ & $1,11 \mathrm{E}+3^{*}$ & $1,32 \mathrm{E}+3^{*}$ & $1,71 \mathrm{E}+3^{\star}$ & $1,22 \mathrm{E}+2^{\star}$ & $1,58 \mathrm{E}+2^{\star}$ & $3,33 E+2^{*}$ & $4,32 \mathrm{E}+2^{\star}$ \\
\hline $\begin{array}{l}\text { Inalação de vapores } \\
\text { em ambientes } \\
\text { fechados a partir da } \\
\text { água subterrânea }\end{array}$ & $\mathrm{mg} / \mathrm{L}$ & 1 & $5,73 E+0$ & $1,83 E+1$ & $7,01 E+0$ & $2,24 \mathrm{E}+1$ & $2,00 E+0$ & $6,40 \mathrm{E}+0$ & $2,60 E+0$ & $8,29 E+0$ \\
\hline $\begin{array}{l}\text { Ingestão de água } \\
\text { subterrânea a partir } \\
\text { da lixiviação do solo } \\
\text { subsuperficial }\end{array}$ & $\mathrm{mg} / \mathrm{kg}$ & 1 & $6,06 \mathrm{E}+0$ & $1,57 E+1$ & $3,11 E+0$ & $8,07 E+0$ & $2,29 E+0$ & $5,94 \mathrm{E}+0$ & $8,95 \mathrm{E}-1$ & $2,32 E+0$ \\
\hline $\begin{array}{l}\text { Contato dérmico } \\
\text { com a água subter- } \\
\text { rânea a partir da } \\
\text { lixiviação do solo } \\
\text { subsuperficial }\end{array}$ & $\mathrm{mg} / \mathrm{kg}$ & 1 & $9,37 \mathrm{E}+0$ & $1,21 E+1$ & $4,81 E+0$ & $6,24 \mathrm{E}+0$ & $2,98 \mathrm{E}+0$ & $3,86 \mathrm{E}+0$ & $1,16 \mathrm{E}+0$ & $1,51 E+0$ \\
\hline
\end{tabular}

*Valores que ultrapassaram os limites físico-químicos de ocorrência do composto no meio avaliado e não devem ser utilizados na avaliação de risco.

água $\left(\mathrm{K}_{\mathrm{oc}}\right)$ apresentados pelos $\mathrm{PAH}$, o que determina baixos fatores de lixiviação e, consequentemente, NABR acima da concentração de saturação do constituinte no meio avaliado. Ainda neste contexto, os PAH possuem baixos valores de pressão de vapor e de constante da Lei de Henry, o que resulta em baixa capacidade de volatilização e mobilidade.

Os resultados de NABR não têm por objetivo constituir valores padrões ou metas de remediação. Entretanto, por serem derivados de parâmetros regionais muitas vezes conservadores, implicam uma necessidade de avançar para um nível mais aprofundado de investigação ambiental e análise de risco. Nesse sentido, as tabelas de referência auxiliam no gerenciamento de áreas potencialmente impactadas, priorizando-se recursos técnico-financeiros em áreas onde os riscos se apresentaram inaceitáveis.

A avaliação comparativa entre os NABR obtidos no presente trabalho e os valores estabelecidos por Maximiano (2001) para a cidade de São Paulo e pela Cetesb (2006) no Estado de São Paulo demonstrou um quadro mais conservador para Porto Alegre, apesar de diferenças nos dados de entrada.

\section{Fatores de incerteza}

Fatores de incerteza podem estar presentes em todas as etapas de avaliação de risco e são inerentes ao processo. Na determinação dos 
NABR deste estudo, incertezas vinculam-se aos parâmetros de exposição, aos dados de toxicidade dos compostos orgânicos selecionados e aos dados do meio físico.

Os parâmetros de exposição adotados para a população do Município de Porto Alegre são aqueles empregados pela Cetesb, considerados representativos para a população do Estado de São Paulo e população brasileira.

Os dados de toxicidade disponíveis são resultados de estudos toxicológicos realizados em animais e, em alguns casos, em seres humanos. No entanto, os compostos selecionados neste trabalho, como tolueno e xilenos, não possuem dados suficientes sobre o potencial carcinogênico. O fator de carcinogenicidade (SF) e a concentração de referência (RfC) são estabelecidos com grande margem de segurança e são valores conservadores. Além disso, os parâmetros de inalação $\left(\mathrm{RfD}_{\mathrm{i}}\right.$ e $\left.\mathrm{SF}_{\mathrm{i}}\right)$ e contato dérmico $\left(\mathrm{RfD}_{\mathrm{ABS}}\right.$ e $\left.\mathrm{SF}_{\mathrm{ABS}}\right)$ são definidos por meio de equações.

A dificuldade em identificar heterogeneidades do meio físico é refletida em incertezas nos resultados de NABR. Portanto, o grau de confiança nos valores calculados está diretamente ligado à representatividade dos dados do meio físico avaliado.

\section{Conclusões}

Tabelas de referência foram geradas utilizando-se a metodologia RBCA na área abrangida pela cidade de Porto Alegre. Dados das duas unidades hidrogeológicas (Embasamento Cristalino alterado e Depósitos Sedimentares Quaternários) foram extraídos de trabalhos anteriores, compreendendo profundidade do nível d'água, gradiente hidráulico, condutividade hidráulica, porosidade total, densidade do solo, fração de carbono orgânico e conteúdo volumétrico de água na zona não saturada.

A análise estatística dos dados do meio físico em Porto Alegre indicou variações pouco significativas entre as duas unidades hidrogeológicas, o que determinou valores de NABR relativamente próximos. As tabelas de referência obtidas expressam, na maioria dos casos, que os resultados são mais restritivos para o Embasamento Cristalino.

Comparando-se os resultados de NABR aos referentes ao Estado de São Paulo, observa-se que as tabelas de referência de Porto Alegre apresentam, em geral, um quadro mais conservador, ou seja, valores de NABR inferiores aos definidos pela Cetesb. Por esta razão, os estudos de áreas contaminadas na referida cidade demandariam investigações ambientais mais detalhadas e avaliações de risco específicas, influenciando no gerenciamento ambiental.

A elaboração das tabelas de referência para o Município de Porto Alegre atingiu os objetivos propostos. $\mathrm{O}$ aperfeiçoamento deste modelo pode ser alcançado por uma rede mais densa de amostragem e realização de estudos que definam parâmetros específicos para a população local.

\section{Agradecimentos}

Ao Conselho Nacional de Desenvolvimento Científico e Tecnológico (CNPq) pela concessão da bolsa de estudos à primeira autora, às empresas Essencis Soluções Ambientais e ESSO Brasileira de Petróleo, bem como à Secretaria Municipal do Meio Ambiente de Porto Alegre pela disponibilização de dados. Aos professores Élvio Giasson e Nelson Caicedo por suas contribuições.

\section{Referências}

AMERICAN SOCIETY FOR TESTING AND MATERIALS (ASTM). ASTM E1739: Standard guide for risk-based corrective action applied at petroleum release sites. Philadelphia. 1995.

COMPANHIA DE TECNOLOGIA DE SANEAMENTO AMBIENTAL (CETESB). Ações Corretivas Baseadas em Risco (ACBR) Aplicadas a Áreas Contaminadas com Hidrocarbonetos Derivados de Petróleo e Outros Combustíveis Líquidos - Procedimentos. São Paulo: CETESB, 2006.

Relatório de Estabelecimento de Valores Orientadores para Solos e Águas Subterrâneas no Estado de São Paulo. São Paulo: CETESB, 2001.

INTEGRATED RISK INFORMATION SYSTEM (IRIS). Banco de dados toxicológicos da USEPA. Disponível em: <http://www.epa.gov/iris/>. Acesso em: out. 2007.

KIRCHHEIM, R.E; MENEGAT, R. Águas subterrâneas e mapa de aqüiferos. In: ATLAS AMBIENTAL DE PORTO ALEGRE (2a ed.). Porto Alegre: Editora da UFRGS, 1999.
MAXIMIANO, A.M.S. Determinação de Níveis aceitáveis no ambiente para hidrocarbonetos utilizando o procedimento de ações corretivas baseadas no risco (RBCA). aplicação para a cidade de São Paulo. 116 f. Dissertação (Mestrado em Recursos Minerais e Hidrogeologia) Universidade de São Paulo, São Paulo, 2001.

RISK ASSESSMENT INFORMATION SYSTEM (RAIS). Banco de dados para avaliações de risco. Disponível em: < http://rais.ornl.gov/cgi-bin/ tox/TOX select? select $=$ nrad $>$. Acesso em: nov. 2007.

SENTELHAS, P.C. et al. BHBRASIL - Balanços Hídricos Climatológicos de 500 Localidades Brasileiras. 1998. Núcleo de Monitoramento Agroclimático. Escola Superior de Agricultura Luiz de Queiroz, Universidade de São Paulo. Disponível em: <http://www.lce.esalq.usp. br/nurma.html>. Acesso em: set. 2007.

UNITED STATES ENVIRONMENTAL PROTECTION AGENCY (USEPA) Preliminary Remediation Goals (PRG) - Region 9. Users' guide and background technical document for USEPA region 9's preliminary 
remediation goals (PRG) Table. 2004A. Disponível em: <http://www. epa.gov/region09/waste/sfund/prg/> . Acesso em: nov. 2007.

Risk Assessment Guidance for Superfund - Volume I - Human Health Evaluation Manual (Parte E, Supplemental Guidance for Dermal Risk Assessment). Document Number: EPA/540/R/99/005. 2004B.

Superfund Chemical Data Matrix (SCDM). 2004C. Disponível em: <http://www.epa.gov/superfund/sites/npl/hrsres/tools/scdm.htm >. Acesso em: abr. 2007.
Soil Screening Guidance: Technical Background Document Tables of Contents. Document Number: EPA/540/R-95/128, July 1996. Disponível em: <http://www.epa.gov/superfund/resources/soil/toc. htm >. Acesso em: abr. 2007.

. Risk Assessment Guidance for Superfund - Volume I - Human Health Evaluation Manual (Parte B, Development of Risk-Based Preliminary Remediation Goals). Document Number: EPA/540/R-92/003. Publication 9285.7-01B. 1991. 